\title{
A Simple and Efficient Procedure for a 2-Pyridones Synthesis under Solvent-Free Conditions
}

\author{
Zahira Kibou $^{1}$, Nawel Cheikh ${ }^{1,2}$, Didier Villemin ${ }^{2 *}$, Noureddine Choukchou-Braham ${ }^{1}$, \\ Bachir Mostefa-Kara ${ }^{1}$, Mohamed Benabdallah ${ }^{1}$ \\ ${ }^{1}$ Laboratoire de Catalyse et Synthèse en Chimie Organique, Faculté des Sciences, Université Aboubekr Belkaïd, \\ Tlemcen, Algérie \\ ${ }^{2}$ ENSICAEN, LCMT, Caen, France \\ E-mail:*didier.villemin@ensicaen.fr
}

Received June 7, 2011; revised July 21, 2011; accepted August 9, 2011

\begin{abstract}
A new series of 3-cyano-2-pyridones derivatives have been prepared by reaction of enaminonitriles with primary amine under solvent free condition. This procedure have the advantage of high yields and being environmentally-friendly.
\end{abstract}

Keywords: 2-Pyridones, Solvent-Free, Enaminonitriles, Green Chemistry

\section{Introduction}

Nowadays, one powerful solution in the Green \& Sustainable Chemistry movement is the replacement of traditional synthetic methods, which use harmful stoichiometric reagents that produce vast amounts of wastes, with clean and simple catalytic alternatives with high atom efficiency $[1,2]$. Solvent-free and dominos reactions represent very powerful green chemical technology procedures from both the economical and synthetic point of view and represent a possible instrument to perform a near-ideal synthesis because they enhance the rate of many organic reactions and afford quantitative yields [3-8]. Heteroaromatic rings containing atoms frequently play an important role as the scaffolds of bioactive substances [9]. It is well-known that the pyridone [9] and its derivatives are among the most popular N-heteroaromatic compounds integrated into the structures of many pharmaceutical compounds and the structural units occur in various molecules exhibiting diverse biological activities [10-12]. This can easily be demonstrated using the following examples (Figure 1) [13]. Pyridone $L-697,661$ [13] has been recognized as a specific non-nucleoside reverse transcriptase inhibitor of human immunodeficiency virus-1 (HIV-1) [13], Milrinone WIN 47203 $[9,14]$, Amrinone WIN $40680[9,14]$ and their analogues are well time- honored positive inotropic and vasodilatatory agents, used in the clinical treatment of heart failure $[9,14]$. Some others are reported to show antitumor [15], antibacterial activity, evaluated as human rhinovirus (HRV) 3C-protease (3CP) inhibitors [15] and other biological activities. Others, that share the 2-Pyridone and its derivatives, illustrate a large class as ligands in coordination chemistry $[16,17]$.

The various research teams around the world were and are still interested in the synthesis of 2-Pyridones (Pyridin-2(1H)-ones). The various synthetic approaches to 2pyridones of this type are described. Many literature sources [18-26] describe more general approaches involving the condensation of unsaturated ketones with methylene active amides, using cyanoacetamide. A number of Milrinone (Figure 1) analogues have been obtained [18-26]. Departing from the previous literature, and as part of our continuing interest in the progress of new synthetic methods in heterocyclic chemistry in our laboratory [27-29], we started the development of a new preparative procedure for this class of heterocyclic scaffold compounds.

\section{Results and Discussions}

In our work, we developed a new method for an easier, simpler and more universal synthesis to prepare this type of heterocycles "2-pyridone", while trying to respect the criteria of the green chemistry, in which we employed, as a key step, the synthesis of enaminonitrile and in the presence of a catalytic amount of $\mathrm{NH}_{4} \mathrm{OAc}$ (Scheme 1).

From Scheme 1, we found that the synthesis under solvent-free of new nitrogen heterocyclic compounds of "2-pyridone derivatives" can be obtained, by a simple, effective, fast and cleaner method, using the three following steps: 


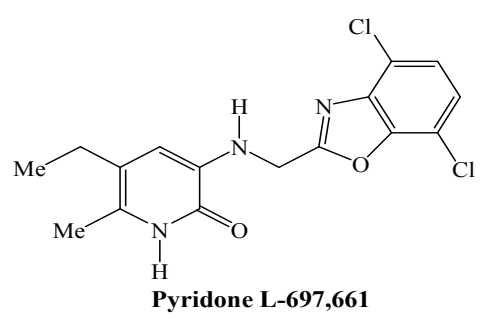

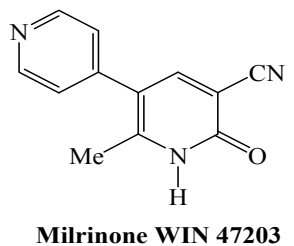<smiles></smiles>

Figure 1. Structures of pyridones L697,661, Milrinone WIN 47203, Amrinone WIN 40680.<smiles>O=C([AlH2])Cl</smiles><smiles>CCOC(=O)C(C#N)=C(C)[AlH2]</smiles><smiles>[R]n1ccc(Br)c(CC2CC3CCC2C3)c1=O</smiles><smiles>CC(C)(C)C1(C)CCCCC1</smiles><smiles>CC#CC</smiles><smiles>CC#CC(=O)C(C#N)=C([Al])C=CN(C)C</smiles>

Scheme 1. Synthesis of 3-cyano-2-pyridone derivatives. Reagents and conditions: (i) $\mathrm{NH}_{4} \mathrm{OAc}, \mathrm{AcOH}, 100^{\circ} \mathrm{C}, 3$ hours, solvent-free, 65\% - 80\%; (ii) DMF-DMA (10 mmol), r.t., solvent-free, 24 hours, 75\% - 90\%; (iii) $\mathrm{RNH}_{2}$ solvent-free, 1 - 4 hours, $48 \%$ - $88 \%$.

\subsection{Knoevenagel Condensation of Acetophenone Derivatives}

The Kknoevenagel condensation is one of the basic necessary reactions in organic chemistry. The research process for this reaction was developed very rapidly. Considering the importance of this condensation, several synthesis methods were carried out. Usually, it is carried out in the presence of harmful organic solvents such as benzene and the DMF [30], and catalysts such as $\mathrm{Al}_{2} \mathrm{O}_{3}$ [31], silica gel [32], a basic ionic liquid [33], $\mathrm{Na}_{2} \mathrm{CO}_{3}-\mathrm{MS} 4$ $\AA$ [34], Mn (III) salen [35], and $\mathrm{NH}_{4} \mathrm{OAc}$-basic alumina [36].

From our side, as a first step, we have prepared a series of ethyl 2-cyano-3-arylbut-2-enoate (2a-f), $\alpha, \beta$-unsaturated compounds, according to the knoevenagel condensation of a sequence of aromatic ketones (1a-f), with of the ethyl cyanoacetate catalyzed by ammonium acetate at $100^{\circ} \mathrm{C}$, under solvent-free conditions (Scheme 1). The ethyl 2-cyano-3-arylbut-2-enoate 2a-f was obtained with a moderate to excellent yields. The results are reported in Table 1.

\subsection{Synthesis of Enaminonitriles}

These olefins, $\alpha, \beta$-unsaturated compounds, prepared by Knoevenagel condensations are largely used as key products in organic syntheses. They found a major applica- tion in medicine, biology, and agriculture; thanks to their Michael acceptor properties [37,38]. Therefore, they are attractive molecules; as they have an exploitable functional richness for organic chemistry, where we were interested in acid methylene, for synthesis of enaminonitriles.

For a long time, many strategies have been considered for the enaminonitriles synthesis [10-12,39]. Promoted by the literature, we prepared the ethyl 2-cyano-5-(dimethylamino)-3-arylpenta-2,4-dienoate 3a-f (enaminonitriles) using the reaction between $\mathbf{2 a - b}$ and $N, N$-dimethylformamide-dimethylacetal (DMF-DMA) under solvent-free, at room temperature (Scheme 1). The yields obtained are very satisfactory 75\% - 90\% (Table 2 ).

\subsection{Synthesis of 3-Cyano-2-Pyridones}

The enaminonitriles are "push-pull" dienes and a good synthon for the organic synthesis, because they can react with the nucleophilic and electrophilic agents. They are used in the preparation of various heterocycles $[39,40]$.

In this last step, well-known as the cyclization step, and in order to study the reactivity of enaminonitriles, we added various types of primary nucleophilic amines to the ethyl 2-cyano-5-(dimethylamino)-3-arylpenta-2,4-dienoate 3a-f (enaminonitrile), under solvent-free (Scheme 1). The mixture was heated, for a few hours, to form 3-cyano-2-pyridone derivatives, with moderate to excellent yields (Table 3). 
Table 1. Solvent-free Knoevenagel condensation for the synthesis of 2a-f.

\begin{tabular}{|c|c|c|c|}
\hline Entry & $\mathrm{Ar}$ & Product & Yield (\%) \\
\hline 1 & $\mathrm{C}_{6} \mathrm{H}_{5^{-}}$ & & 70 \\
\hline 2 & $p-\mathrm{ClC}_{6} \mathrm{H}_{4^{-}}$ & & 75 \\
\hline 3 & $2,4-\mathrm{Cl}_{2} \mathrm{C}_{6} \mathrm{H}_{3}-$ & & 68 \\
\hline 4 & $m-\mathrm{CH}_{3} \mathrm{OC}_{6} \mathrm{H}_{4}-$ & & 73 \\
\hline 5 & $p-\mathrm{CH}_{3} \mathrm{OC}_{6} \mathrm{H}_{4}-$ & & 80 \\
\hline 6 & $p-\mathrm{CH}_{3} \mathrm{C}_{6} \mathrm{H}_{4^{-}}$ & & 65 \\
\hline
\end{tabular}

Table 2. Synthesis of enaminonitrile (3a-f) without solvent.

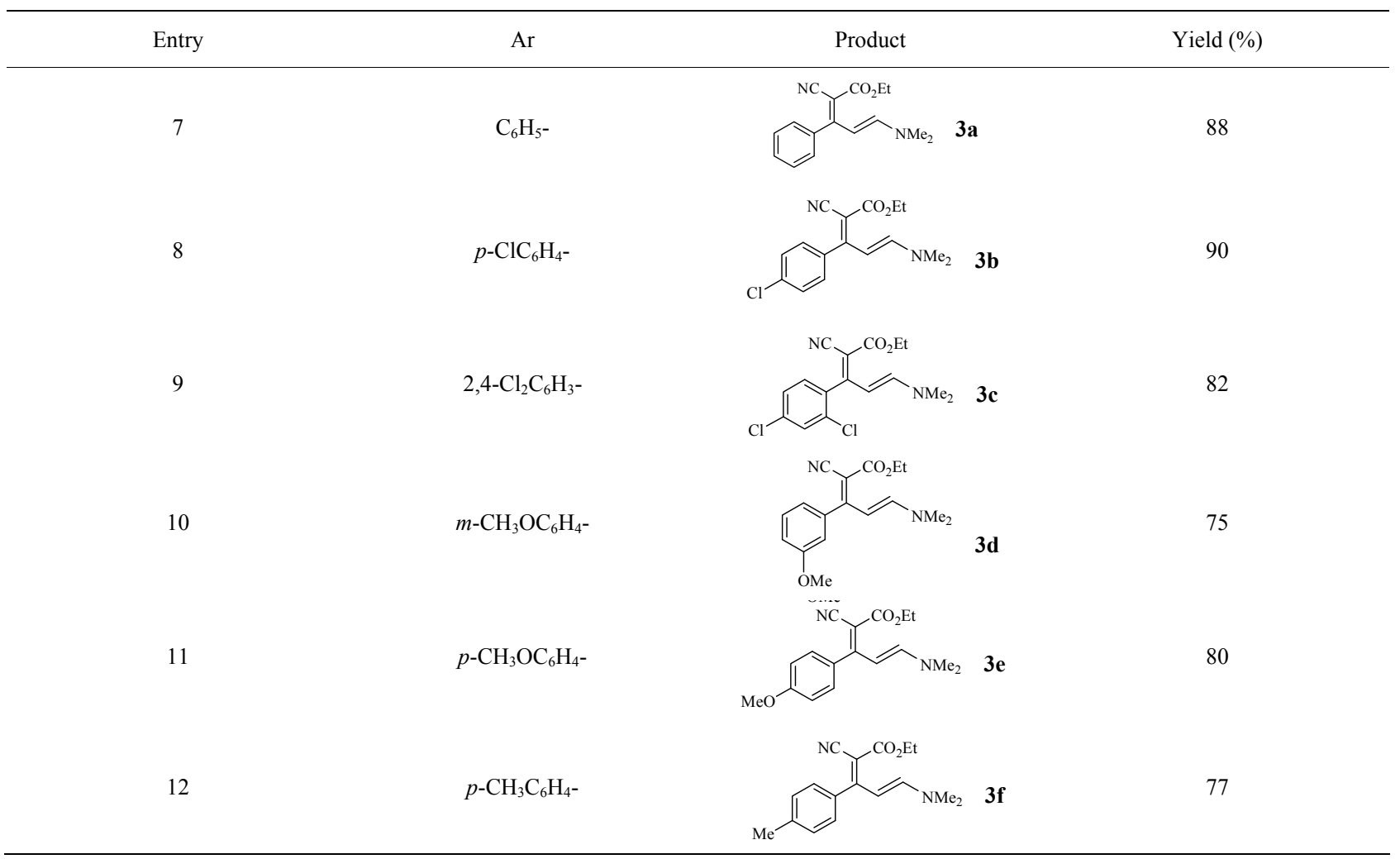


Table 3. Synthesis of the 3-cyano-2-pyridones.

\begin{tabular}{|c|c|c|c|c|c|}
\hline Entry & Enaminonitrile & $\mathrm{R}$ & Product & & Yield (\%) \\
\hline 13 & & $\mathrm{CH}_{3}-$ & & $4 a_{1}$ & 66 \\
\hline 14 & & $\mathrm{CH}_{2}=\mathrm{CH}-\mathrm{CH}_{2}-$ & & $4 a_{2}$ & 70 \\
\hline 15 & & $\mathrm{C}_{6} \mathrm{H}_{5} \mathrm{CH}_{2-}$ & & $4 a_{3}$ & 73 \\
\hline 16 & & $\left(\mathrm{CH}_{3}\right)_{2} \mathrm{CH}-$ & & $4 a_{4}$ & 48 \\
\hline 17 & & $\mathrm{CH}_{3^{-}}$ & & $4 b_{1}$ & 76 \\
\hline 18 & & $\mathrm{CH}_{2}=\mathrm{CH}-\mathrm{CH}_{2}-$ & & $4 \mathbf{b}_{2}$ & 80 \\
\hline 19 & & $\mathrm{C}_{6} \mathrm{H}_{5} \mathrm{CH}_{2-}$ & & & 85 \\
\hline 20 & & $\left(\mathrm{CH}_{3}\right)_{2} \mathrm{CH}-$ & & $4 \mathrm{~b}$ & 50 \\
\hline 21 & & $\mathrm{CH}_{3-}$ & & $4 c_{1}$ & 75 \\
\hline 22 & & $\mathrm{CH}_{2}=\mathrm{CH}-\mathrm{CH}_{2}-$ & & $4 c_{2}$ & 80 \\
\hline 23 & & $\mathrm{C}_{6} \mathrm{H}_{5} \mathrm{CH}_{2^{-}}$ & & & 84 \\
\hline 24 & & $\left(\mathrm{CH}_{3}\right)_{2} \mathrm{CH}-$ & & $4 c_{4}$ & 52 \\
\hline 25 & & $\mathrm{CH}_{3-}$ & & $\mathbf{4 d _ { 1 }}$ & 73 \\
\hline 26 & & $\mathrm{CH}_{2}=\mathrm{CH}-\mathrm{CH}_{2}-$ & & $4 d_{2}$ & 79 \\
\hline 27 & & $\mathrm{C}_{6} \mathrm{H}_{5} \mathrm{CH}_{2-}$ & & & 80 \\
\hline 28 & & $\left(\mathrm{CH}_{3}\right)_{2} \mathrm{CH}-$ & & & 53 \\
\hline
\end{tabular}




(2)

\section{Conclusions}

In summary, we have developed a simple, efficient and rapid method for the synthesis of 3-cyano-2-pyridones, following three steps, i.e. the Knoevenagel condensation catalyzed by $\mathrm{NH}_{4} \mathrm{OAc}$, the enaminonitriles synthesis, and finally the synthesis of the 3-cyano-2-pyridone under solvent-free conditions. This procedure has the advantages of being a mild conditions reaction, using a catalytic quantity of $\mathrm{NH}_{4} \mathrm{OAc}$, with moderate to excellent yields, and where we operate with simplicity while respecting the criteria of Green Chemistry.

\section{Experimental}

The melting points were measured using a Bank Kofler HEIZBANK apparatus standard WME $50-260^{\circ} \mathrm{C}$ and were uncorrected. IR spectra were obtained with solids with a Fourier transform Perkin Elmer Spectrum One with ATR accessory. Only significant absorptions are listed. The ${ }^{1} \mathrm{H}$ NMR spectra were recorded at $400 \mathrm{MHz}$, on a Brüker AC 400 spectrometers and ${ }^{13} \mathrm{C}$ NMR spectra were recorded in the same spectrometers at 100.6 MHz. Samples were registered in $\mathrm{CDCl}_{3}$ solutions using TMS as an internal standard. The chemical shifts are expressed in $\delta$ units (ppm) and quoted downfield from TMS. The multiplicities are reported as: s, singlet; $d$, doublet; t, triplet; q, quartet; $\mathrm{m}$, multiplet.

General procedure 1: Synthesis of ethyl 2-cyano-3(aryl) but-2-enoate 2a-b

A mixture of acetophenone or substituted acetophe- none $(10 \mathrm{mmol})$, ethyl cyanoacetate $(10 \mathrm{mmol})$, ammonium acetate $(10 \mathrm{mmol})$ and some drops of icy acetic acid were stirred and heated at $100^{\circ} \mathrm{C}$ during 3 hours. The reaction mixture was cooled down to room temperature, diluted with $30 \mathrm{ml}$ of $\mathrm{CH}_{2} \mathrm{Cl}_{2}$. The organic layer obtained was washed with $(3 \times 20 \mathrm{ml})$ of water, $(10 \mathrm{ml})$ of saturated $\mathrm{NaCl}$, dried on $\mathrm{MgSO}_{4}$, filtered then evaporated under vacuum. The compounds $\mathbf{2 a - f}$ were obtained as colourless oil.

Ethyl 2-cyano-3-phenylbut-2-enoate 2a. The general procedure 1 , using $(1.20 \mathrm{~g}, 10 \mathrm{mmol})$ of acetophenone, $(1.13 \mathrm{~g}, 10 \mathrm{mmol})$ of ethyl cyanoacetate, $(0.77 \mathrm{~g}, 10$ $\mathrm{mmol}$ ) of ammonium acetate and some drops of icy acetic acid, gave $70 \%$ of $\mathbf{2 a}$ as burn oil. ${ }^{1} \mathrm{H}$ NMR $(400 \mathrm{MHz}$, $\left.\mathrm{CDCl}_{3}\right): 7.53-7.14(\mathrm{~m}, 5 \mathrm{H}), 4.20\left(\mathrm{q}, 2 \mathrm{H}, J_{\mathrm{H}-\mathrm{H}}=7.2 \mathrm{~Hz}\right)$, $2.57(\mathrm{~s}, 3 \mathrm{H}), 1.26\left(\mathrm{t}, 3 \mathrm{H}, J_{\mathrm{H}-\mathrm{H}}=7.2 \mathrm{~Hz}\right) \cdot{ }^{13} \mathrm{C}$ NMR $(100$ $\left.\mathrm{MHz}, \mathrm{CDCl}_{3}\right): 172.21,162.12,136.91-128.10,116.01$, 104.95, 61.83, 24.50, 13.75. IR (neat, $\mathrm{cm}^{-1}$ ): 2225,1747 , 1682.

Ethyl 3-(4-chlorophenyl)-2-cyanobut-2-enoate 2b. The general procedure 1 , using $(1.54 \mathrm{~g}, 10 \mathrm{mmol})$ of 4-chloroacetophenone, $(1.13 \mathrm{~g}, 10 \mathrm{mmol})$ of ethyl cyanoacetate and $(0.77 \mathrm{~g}, 10 \mathrm{mmol})$ of ammonium acetate and some drops of icy acetic acid, gave $75 \%$ of $\mathbf{2 b}$ as burn oil. ${ }^{1} \mathrm{H}$ NMR (400 MHz, $\left.\mathrm{CDCl}_{3}\right): 7.51-7.18(\mathrm{~m}, 4 \mathrm{H}), 4.20$ (q, $\left.2 \mathrm{H}, J_{\mathrm{H}-\mathrm{H}}=7.2 \mathrm{~Hz}\right), 2.62(\mathrm{~s}, 3 \mathrm{H}), 1.29\left(\mathrm{t}, 3 \mathrm{H}, J_{\mathrm{H}-\mathrm{H}}=7.2\right.$ $\mathrm{Hz}) .{ }^{13} \mathrm{C}$ NMR $\left(100 \mathrm{MHz}, \mathrm{CDCl}_{3}\right): 174.81,164.23$, 136.75 - 128.60, 115.38, 102.00, 62.10, 24.50, 24.75. IR (neat, $\mathrm{cm}^{-1}$ ): 2222, 1747, 1682 . 
General procedure 2: Synthesis of ethyl 2-cyano-5(dimethylamino)-3-arylpenta-2, 4-dienoate 3a-b

A mixture of ethyl 2-cyano-3-arylbut-2-enoate (10 mmol) 2a-b, $N, N$-dimethylformamide dimethyl acetal (10 $\mathrm{mmol}$ ) were stirred at room temperature without solvent during 24 hours. The solution takes a colouring increasingly dark burn. The purple solid obtained was washed several times with diethyl ether and crystallised in absolute ethanol to provide products $\mathbf{3 a - b}$.

Ethyl 2-cyano-5-(dimethylamino)-3-phenylpenta-2, 4-dienoate 3a. The general procedure 2 , using (2.15 g, 10 $\mathrm{mmol})$ of $2 \mathrm{a}$ and $(1.19 \mathrm{~g}, 10 \mathrm{mmol})$ of $N, N$-dimethylformamide dimethyl acetal, gave $85 \%$ of compound 3a as yellow solid, mp $142^{\circ} \mathrm{C} .{ }^{1} \mathrm{H}$ NMR (400 MHz, $\left.\mathrm{CDCl}_{3}\right): 7.32-7.25(\mathrm{~m}, 5 \mathrm{H}), 6.57\left(\mathrm{~d}, 1 \mathrm{H}, J_{\mathrm{H}-\mathrm{H}}=12.8 \mathrm{~Hz}\right)$, $5.98\left(\mathrm{~d}, 1 \mathrm{H}, J_{\mathrm{H}-\mathrm{H}}=12.8 \mathrm{~Hz}\right), 4.32\left(\mathrm{q}, 2 \mathrm{H}, J_{\mathrm{H}-\mathrm{H}}=7.2 \mathrm{~Hz}\right)$, $3.07(\mathrm{~s}, 3 \mathrm{H}), 3.04(\mathrm{~s}, 3 \mathrm{H}), 1,39\left(\mathrm{t}, 3 \mathrm{H}, J_{\mathrm{H}-\mathrm{H}}=7.2 \mathrm{~Hz}\right),{ }^{13} \mathrm{C}$ NMR $\left(100 \mathrm{MHz}, \mathrm{CDCl}_{3}\right): 169.41,165.56,156.71$, 137.80 - 127.70, 119.88, 99.75, 86.04, 60.07, 45.43, 37.48, 14.37. IR (neat, $\mathrm{cm}^{-1}$ ): 2191, 1674, 1609, 1508.

Ethyl 3-(4-chlorophenyl)-2-cyano-5-(dimethy-lamino) penta-2,4-dienoate $3 \mathrm{~b}$. The general procedure 2 , using ( $2.49 \mathrm{~g}, 10 \mathrm{mmol})$ of $\mathbf{2 b}$, and $(1.19 \mathrm{~g}, 10 \mathrm{mmol})$ of $\mathrm{N}, \mathrm{N}$-dimethylformamide dimethyl acetal, gave $90 \%$ of compound $\mathbf{3 b}$ as yellow solid, mp $208^{\circ} \mathrm{C} .{ }^{1} \mathrm{H}$ NMR (400 $\left.\mathrm{MHz}, \mathrm{CDCl}_{3}\right): 7.35-7.20(\mathrm{~m}, 4 \mathrm{H}), 6.48\left(\mathrm{~d}, 1 \mathrm{H}, J_{\mathrm{H}-\mathrm{H}}=\right.$ $12.8 \mathrm{~Hz}), 5,90\left(\mathrm{~d}, 1 \mathrm{H}, J_{\mathrm{H}-\mathrm{H}}=12.8 \mathrm{~Hz}\right), 4.26\left(\mathrm{q}, 2 \mathrm{H}, J_{\mathrm{H}-\mathrm{H}}\right.$ $=7.2 \mathrm{~Hz}$,), $3.01(\mathrm{~s}, 3 \mathrm{H}), 2.99(\mathrm{~s}, 3 \mathrm{H}), 1.33\left(\mathrm{t}, 3 \mathrm{H}, J_{\mathrm{H}-\mathrm{H}}=\right.$ $7.2 \mathrm{~Hz}) .{ }^{13} \mathrm{C}$ NMR $\left(100 \mathrm{MHz}, \mathrm{CDCl}_{3}\right): 169.88,158.38$, $140.75,137.20$ - 128.75, 115.41, 107, 89.08, 60.01, 47.80, 38.77, 14.28. IR (neat, $\mathrm{cm}^{-1}$ ): 2199, 1680, 1604, 1507.

General procedure 3: Synthesis of 2-Pyridones $4 a_{i}-b_{i}$

A mixture of ethyl 2-cyano-5-(dimethylamino)-3-arylpenta-2,4-dienoate 3a-b $(2 \mathrm{mmol})$ and primary amine ( $2 \mathrm{mmol}$ ) were heated for a few hours. After cooling, the solid obtained was washed several times with diethyl ether to give 2-pyridone derivatives $\mathbf{4} \mathbf{a}_{\mathbf{i}}-\mathbf{b}_{\mathbf{i}}$.

1,2-dihydro-1-methyl-2-oxo-4-phenylpyridine-3-car bonitrile $4 \mathrm{a}_{1}$. The general procedure 3 , using $(0.43 \mathrm{~g}, 2$ $\mathrm{mmol})$ of 3a and (0.06 g, $2 \mathrm{mmol})$ of methylamine, gave $66 \%$ of compound $4 \mathrm{a}_{1}$ as white solid, mp $174^{\circ} \mathrm{C} .{ }^{1} \mathrm{H}$ NMR (400 MHz, $\left.\mathrm{CDCl}_{3}\right): 7.55\left(\mathrm{~d}, 1 \mathrm{H}, J_{\mathrm{H}-\mathrm{H}}=6.7 \mathrm{~Hz}\right)$, $7.51-7.50(\mathrm{~m}, 5 \mathrm{H}), 6.62\left(\mathrm{~d}, 1 \mathrm{H}, J_{\mathrm{H}-\mathrm{H}}=6.4 \mathrm{~Hz}\right), 3.62(\mathrm{~s}$, $3 \mathrm{H}) .{ }^{13} \mathrm{C}$ NMR (100 MHz, $\left.\mathrm{CDCl}_{3}\right): 159.82,158.87$, 134.46, 129.69 - 127.00, 114.57, 106.04, 101.42, 37.12. IR (neat, $\mathrm{cm}^{-1}$ ): 2220, 1645, 1597.

1-allyl-1,2-dihydro-2-oxo-4-phenylpyridine-3-carbo nitrile $4 \mathbf{a}_{2}$. The general procedure 3 , using $(0.43 \mathrm{~g}, 2$ $\mathrm{mmol}) \mathbf{3 a}$ and $(0.11 \mathrm{~g}, 2 \mathrm{mmol})$ of allylamine, gave $76 \%$ of compound $\mathbf{4 a}_{2}$ as white solid, mp $99^{\circ} \mathrm{C}-100^{\circ} \mathrm{C} .{ }^{1} \mathrm{H}$ NMR (400 MHz, $\left.\mathrm{CDCl}_{3}\right): 7.61(\mathrm{~d}, 1 \mathrm{H}), 7.53$ - $7.49(\mathrm{~m}$, $5 \mathrm{H}), 6.36\left(\mathrm{~d}, 1 \mathrm{H}, J_{\mathrm{H}-\mathrm{H}}=6.7 \mathrm{~Hz}\right), 6.02-5.92(\mathrm{~m}, 1 \mathrm{H})$,
5.37 - $5.30(\mathrm{~m}, 2 \mathrm{H}), 4.64-4.62(\mathrm{~m}, 2 \mathrm{H}) .{ }^{13} \mathrm{C}$ NMR $(100$ $\left.\mathrm{MHz}, \mathrm{CDCl}_{3}\right): 160.2,159.78,140.41,135.46,131.29-$ $128.03,120.29,115.55,107.26,102.76,51.26$. IR (neat, $\left.\mathrm{cm}^{-1}\right): 2216,1634,1591$.

1-benzyl-1,2-dihydro-2-oxo-4-phénylpyridine-3-car bonitrile $4 \mathrm{a}_{3}$. The general procedure 3 , using $(0.43 \mathrm{~g}, 2$ $\mathrm{mmol})$ of $3 \mathbf{a}$ and $(0.21 \mathrm{~g}, 2 \mathrm{mmol})$ of benzylamine, gave $73 \%$ of compound $4 \mathrm{a}_{3}$ as white solid, mp $134^{\circ} \mathrm{C} .{ }^{1} \mathrm{H}$ NMR (400 MHz, $\left.\mathrm{CDCl}_{3}\right): 7.60\left(\mathrm{~d}, 1 \mathrm{H}, J_{\mathrm{H}-\mathrm{H}}=7.2 \mathrm{~Hz}\right)$, $7.58-7.38(\mathrm{~m}, 2 \times 5 \mathrm{H}), 6.31\left(\mathrm{~d}, 1 \mathrm{H}, J_{\mathrm{H}-\mathrm{H}}=7.2 \mathrm{~Hz}\right), 5.19$ (s, $2 \mathrm{H}) \cdot{ }^{13} \mathrm{C}$ NMR $\left(100 \mathrm{MHz}, \mathrm{CDCl}_{3}\right): 160.55,159.66$, $140.44,135.43,134.96$ - 128.71, 128.03, 115.98, 107.36, 52.69. IR (neat, $\mathrm{cm}^{-1}$ ): 2220, 1645, 1597.

1,2-dihydro-1-isopropyl-2-oxo-4-phenylpyridine-3carbonitrile $\mathbf{4 a}_{4}$. The general procedure 3 , using $(0.43 \mathrm{~g}$, $2 \mathrm{mmol})$ of $3 \mathbf{a}$ and of $(0.11 \mathrm{~g}, 2 \mathrm{mmol})$ isopropylamine gave $48 \%$ of compound $4 \mathbf{a}_{4}$ as withe solid, mp $144^{\circ} \mathrm{C} .{ }^{1} \mathrm{H}$ NMR $\left(400 \mathrm{MHz}, \mathrm{CDCl}_{3}\right): 7.57\left(\mathrm{~d}, 1 \mathrm{H}, J_{\mathrm{H}-\mathrm{H}}=6.8 \mathrm{~Hz}\right)$, $7.49-7.50(\mathrm{~m}, 5 \mathrm{H}), 6.38\left(\mathrm{~d}, 1 \mathrm{H}, J_{\mathrm{H}-\mathrm{H}}=6.8 \mathrm{~Hz}\right), 5.24-5.31$ $(\mathrm{m}, 1 \mathrm{H}), 1.42\left(\mathrm{~d}, 6 \mathrm{H}, J_{\mathrm{H}-\mathrm{H}}=7.2 \mathrm{~Hz}\right) .{ }^{13} \mathrm{C} \mathrm{NMR}(100 \mathrm{MHz}$, $\left.\mathrm{CDCl}_{3}\right): 160.274,158.80,136.85,135.56$ - 127.57, 115.86, 107.01, 102.34, 47.68, 21.78. IR (neat, $\mathrm{cm}^{-1}$ ): 2219, 1640, 1592, 1517.

4-(4-chlorophenyl)-1-methyl-2-oxo-1,2-dihydropyri dine-3-carbonitrile $4 b_{1}$. The general procedure 3 , using $(0.60 \mathrm{~g}, 2 \mathrm{mmol})$ of $\mathbf{3 b}$ and $(0.06 \mathrm{~g}, 2 \mathrm{mmol})$ of methylamine, gave $76 \%$ of compound $\mathbf{4} \mathbf{b}_{1}$ as white solid, $\mathrm{mp}$ $184^{\circ} \mathrm{C} .{ }^{1} \mathrm{H}$ NMR $\left(400 \mathrm{MHz}, \mathrm{CDCl}_{3}\right): 7.55\left(\mathrm{~d}, 1 \mathrm{H}, J_{\mathrm{H}-\mathrm{H}}=\right.$ $6.7 \mathrm{~Hz}), 7.54-7.47(\mathrm{~m}, 4 \mathrm{H}), 6.30\left(\mathrm{~d}, 1 \mathrm{H}, J_{\mathrm{H}-\mathrm{H}}=6.4 \mathrm{~Hz}\right)$, $3.64(\mathrm{~s}, 3 \mathrm{H}) .{ }^{13} \mathrm{C}$ NMR $\left(100 \mathrm{MHz}, \mathrm{CDCl}_{3}\right): 162.12$, 157.66, 135.56, 127.33 - 129.88, 115.08, 107.80, 103.34, 34.20. IR (neat, $\mathrm{cm}^{-1}$ ): 2221, 1644, 1599 .

1-allyl-4-(4-chlorophenyl)-2-oxo-1,2-dihydropyridi ne-3-carbonitrile $\mathbf{4} \mathbf{b}_{2}$. The general procedure 3 , using $(0.60 \mathrm{~g}, 2 \mathrm{mmol})$ of $\mathbf{3 b}$ and $(0.11 \mathrm{~g}, 2 \mathrm{mmol})$ of allylamine, gave $80 \%$ of compound $\mathbf{4} \mathbf{b}_{2}$ as white solid, mp $172^{\circ} \mathrm{C} .{ }^{1} \mathrm{H}$ NMR $\left(400 \mathrm{MHz}, \mathrm{CDCl}_{3}\right): 7.62(\mathrm{~d}, 1 \mathrm{H})$, $7.56-7.41(\mathrm{~m}, 4 \mathrm{H}), 6.44\left(\mathrm{~d}, 1 \mathrm{H}, J_{\mathrm{H}-\mathrm{H}}=6.7 \mathrm{~Hz}\right), 5.93-$ $5.58(\mathrm{~m}, 1 \mathrm{H}), 5.34-5.28(\mathrm{~m}, 2 \mathrm{H}), 4.76-4.68(\mathrm{~m}, 2 \mathrm{H})$. ${ }^{13} \mathrm{C}$ NMR $\left(100 \mathrm{MHz}, \mathrm{CDCl}_{3}\right)$ : 162.11, 160.22, 143.11, $136.80,134.2$ - 126.50, 122.18, 115.9, 107.80, 103.11, 48.02. IR (neat, $\mathrm{cm}^{-1}$ ): 2216, 1637, 1595.

4-(4-chlorophenyl)-1-ethyl-2-oxo-1,2-dihydropyridi ne-3-carbonitrile $\mathbf{4} \mathbf{b}_{3}$. The general procedure 3 , using $(0.60 \mathrm{~g}, 2 \mathrm{mmol})$ of $\mathbf{3 b}$ and $(0.21 \mathrm{~g}, 2 \mathrm{mmol})$ of benzylamine gave $85 \%$ of compound $4 \mathbf{b}_{3}$ as white solid, $\mathrm{mp}$ $214^{\circ} \mathrm{C} .{ }^{1} \mathrm{H}$ NMR $\left(400 \mathrm{MHz}, \mathrm{CDCl}_{3}\right): 7.62(\mathrm{~d}, 1 \mathrm{H}), 7.49-$ $7.30(\mathrm{~m}, 4 \mathrm{H}), 7.29-7.19(\mathrm{~m}, 5 \mathrm{H}), 6.20(\mathrm{~d}, 1 \mathrm{H}), 5.12(\mathrm{~s}$, $2 \mathrm{H}) .{ }^{13} \mathrm{C}$ NMR $\left(100 \mathrm{MHz}, \mathrm{CDCl}_{3}\right): 167.98,156.50$, 136.67, 135.10, 130.29 - 128.27, 119.84, 100.70, 45.63. IR (neat, $\mathrm{cm}^{-1}$ ): 2219, 1656, 1597.

4-(4-chlorophenyl)-1-isopropyl-2-oxo-1,2-dihydrop yridine-3-carbonitrile $\mathbf{4} \mathbf{b}_{4}$. The general procedure 3 , 
using $(0.60 \mathrm{~g}, 2 \mathrm{mmol})$ of $\mathbf{3 b}$ and $(0.11 \mathrm{~g}, 2 \mathrm{mmol})$ of isopropylamine, gave $50 \%$ of compound $4 \mathbf{b}_{4}$ as white solid, mp $142^{\circ} \mathrm{C} .{ }^{1} \mathrm{H}$ NMR $\left(400 \mathrm{MHz}, \mathrm{CDCl}_{3}\right): 7.52(\mathrm{~d}$, $\left.1 \mathrm{H}, J_{\mathrm{H}-\mathrm{H}}=6.8 \mathrm{~Hz}\right), 7.50-7.40(\mathrm{~m}, 4 \mathrm{H}), 6.27\left(\mathrm{~d}, 1 \mathrm{H}, J_{\mathrm{H}-\mathrm{H}}\right.$ $=6.8 \mathrm{~Hz}), 5.24-5.17(\mathrm{~m}, 1 \mathrm{H}), 1.29\left(\mathrm{~d}, 6 \mathrm{H}, J_{\mathrm{H}-\mathrm{H}}=7.2\right.$ $\mathrm{Hz}) .{ }^{13} \mathrm{C}$ NMR (100 MHz, $\left.\mathrm{CDCl}_{3}\right): 163.32,159.18$, $134.88,136.42$ - 127.80, 115.9, 106.08, 101.54, 48.78, 21.26. IR (neat, $\mathrm{cm}^{-1}$ ): 2221, 1648, 1599, 1512.

\section{References}

[1] D. Blasco-Jiménez, A. J. Lopez-Peinado, R. M. MartinAranda, M. Ziolek and I. Sobczak, "Sonocatalysis in Solvent-Free Conditions: An Efficient Eco-Friendly Methodology to Prepare N-Alkyl Imidazoles Using AminoGrafted NbMCM-41," Catalysis Today, Vol. 142, No. 3-4, 2009, pp. 283-287.

doi:10.1016/j.cattod.2008.11.028

[2] D. Kumar, V. B. Reddy, B. G. Mishra, R. K. Ranna, M. N. Nadagouda and R. S. Varma, "Nanosized Magnesium Oxide as Catalyst for the Rapid and Green Synthesis of Substituted 2-Amino-2-Chromenes," Tetrahedron, Vol. 63, No. 15,2007 , pp. 3093-3097. doi:10.1016/j.tet.2007.02.019

[3] L. F. Teitze, "Domino Reactions in Organic Synthesis," Chemical Reviews, Vol. 96, No. 1, 1996, pp. 115-136. doi: $10.1021 / \mathrm{cr} 950027 \mathrm{e}$

[4] R. Maggi, R. Ballini and G. Sartori, "Basic Alumina Catalysed Synthesis of Substituted 2-Amino-2-chromenes via Three-Component Reaction," Tetrahedron Letters, Vol. 45, No. 11, 2004, pp.2297-2299. doi:10.1016/j.tetlet.2004.01.115

[5] J. A. Wang, X. Bokhimi, O. Novaro, T. Lopez, F. Tzompantzi, R. Gomez, J. Navarrete, M. E. Llanos and M. Lopez-Salinas, "Effects of Structural Defects and Acid-Basic Properties on the Activity and Selectivity of Isopropanol Decomposition on Nanocrystallite Sol-Gel Alumina Catalyst," Journal of Molecular Catalysis A: Chemical, Vol. 137, No. 1-3, 1999, pp. 239-256. doi:10.1016/S1381-1169(98)00077-6

[6] S. Paul, P. Nanda, R. Gupta and A. Loupy, “Ac $\mathrm{A}_{2} \mathrm{O}-\mathrm{Py} /$ Basic Alumina as a Versatile Reagent for Acetylations in Solvent-Free Conditions under Microwave Irradiation," Tetrahedron Letters, Vol. 43, No. 23, 2002, pp. 42614265. doi:10.1016/S0040-4039(02)00732-3

[7] C. M. Figueiredo, "Preparation of Aluminas from a Basic Aluminium Carbonate," Catalysis Today, Vol. 5, 1989, pp. 433-442. doi:10.1016/0920-5861(89)80007-0

[8] S. Carre, B. Tapin, N. S. Gnep, R. Revel and P. Magnoux, "Model Reactions as Probe of the Acid-Base Properties of Aluminas: Nature and Strength of Active sites. Correlation with Physicochemical Characterization," Applied Catalysis A: General, Vol. 372, No. 1, 2010, pp. 26-33. doi:10.1016/i.apcata.2009.10.005

[9] M. T. Cocco, C. Congiu and V. Onnis, "Synthesis and Antitumor Activity of 2-Hydroxy-2-pyridones Derivatives," European Journal of Medicinal Chemistry, Vol.
35 , No. 5, 2000, pp. 545-552. doi:10.1016/S0223-5234(00)00149-5

[10] F. Manna, F. Chimenti, A. Bolasco, A. Filippelli and E. Lampa, "Antiinflammatory, Analgesic and Antipyfuztic 4,6-Disubstituted 3-Cyanopyridine-2-ones and 3-Cyano2-aminopyridines," Pharmacological Research, Vol. 26, Suppl. 1, 1992, pp. 267-277. doi:10.1016/1043-6618(92)91243-A

[11] R. L. Parreira, O. Abrahão and S. E. Galembeck, "Conformational Preferences of Non-Nucleoside HIV-1 Reverse Transcriptase Inhibitors," Tetrahedron, Vol. 57, No. 16, 2001, pp. 3243-3253. doi:10.1016/S0040-4020(01)00193-4

[12] X. Fan, D. Feng, Y. Qu, X. Zhang, J. Wang, P. M. Loiseau, G. Andrei, R. Snoeck, E. De Clercq, "Practical and Efficient Synthesis of Pyrano[3,2-c]pyridone, Pyrano [4,3-b]pyran and Their Hybrids with Nucleoside as Potential Antiviraland Antileishmanial Agents," Bioorganic \& Medicinal Chemistry Letters, Vol. 20, No. 3, 2010, pp. 809-813. doi:10.1016/j.bmcl.2009.12.102

[13] E. L. Presti, R. Boggia, A. Feltrin, G. Menozzi, P. Dorigo and L. Mosti, "3-Acetyl-5-acylpyridin-2(1H)-ones and 3Acetyl-7,8-dihydro-2,5(1H,6H)-quinolinediones: Synthesis, Cardiotonic Activity and Computational Studies," II Farmaco, Vol. 54, No. 7, 1999, pp. 465-447. doi:10.1016/S0014-827X(99)00053-1

[14] W. K. Anderson, D. C. Dean and T. Endo, "Synthesis, Chemistry, and Antineoplastic Activity of $\alpha$-Halopyridinium Salts: Potential Pyridone Prodrugs of Acylated Vinylogous Carbinolamine Tumor Inhibitors," Journal of Medicinal Chemistry, Vol. 33, No. 6, 1990, pp. 16671675. doi:10.1021/jm00168a021

[15] P. S. Dragovich, T. J Prins, Z. Ru, E. L. Brown, F. C. Maldonado, S. A. Fuhrman, L. S. Zalman, L. Iuniland, C. A. Lee and S. T. Worland, "Structure-Based design, Synthesis, and Biological Evaluation of Irreversible $\mathrm{Hu}$ man Rhinovirus 3C Protease Inhibitors. 6. Struc-tureActivity Studies of Orally Bioavailable, 2-Pyridone- Containing Peptidomimetics," Journal of Medicinal Chemistry, Vol. 45, No. 8, 2002, pp. 1607-1623. doi:10.1021/jm010469k

[16] B. Kozlevcar, M. Radisek, Z. Jaglicic, F. Merzel, L. Glazar, A. Golobic and P. Segedin, "Strong Antiferromagnetism in the Dinuclear 2-Pyridone Complex with N-C-O Bridges: A Paddle-Wheel Analogue of the Dinuclear Tetracarboxylates," Polyhedron, Vol. 26, 2007, pp. 5414-5419. doi:10.1016/j.poly.2007.08.019

[17] T. Mochida, M. Ueda, C. Aoki and H. Mori, "Structures and Properties of Trans-Dichloro\{Tetrakis (5-Chloro-2 (1H)-pyridone- $O)\} \mathrm{M}(\mathrm{II})[\mathrm{M}=\mathrm{Mn}, \mathrm{Fe}, \mathrm{Co}, \mathrm{Ni}, \mathrm{Cu}]$; Formation of Quasi-Macrocyclic Metal Complexes through Hydrogen Bonding," Inorganica Chimica Acta, Vol. 335, No. 27, 2002, pp. 151-155. doi:10.1016/S0020-1693(02)00817-4

[18] K. R. Gibson, L. Hitzel, R. J. Mortishire-Smith, U. Gerhard, R.A. Jelley, A. J. Reeve, M. Rowley, A. Nadin and A. P. Owens, "Synthesis and Conformational Dynamics of Tricyclic Pyridones Containing a Fused Seven- 
Membered Ring," Journal of Organic Chemistry, Vol. 67, No. 26, 2002, pp. 9354-9360.

doi:10.1021/jo026411a

[19] I. Collins, C. Moyes, et al., "3-Heteroaryl-2-pyridones: Benzodiazepine Site Ligands with Functional Delectivity for Alpha 2/Alpha 3-Subtypes of Human GABA (A) Receptor-Ion Channels," Journal of Medicinal Chemistry, Vol. 45, No. 9, 2002, pp. 1887-1990. doi:10.1021/jm0110789

[20] F. A. Abu-Shanab, A. D. Redhouse, J. R. Thompson and B. J. Wakefield, "Synthesis of 2,3,5,6-Tetrasubtituted Pyridines from Enamines Derived from N, N-Dimethylformamide Dimethyl Acetal," Synthesis, Vol. 5, 1995, pp. 557-560. doi:10.1055/s-1995-3954

[21] W. D. Jones, R. A. Schnettler and E. W. Huber, "A Convenient Synthesis of 5-Acyl-6-substituted 3-cyano-2 (1H)-pyridinones," Journal of Heterocyclic Chemistry, Vol. 27, No. 3, 1990, pp. 511-518. doi:10.1002/jhet.5570270307

[22] H. Fukatsu, Y. Kato, S. Murase and S. Nakagawa, "Synthesis and Cardiotonic Activity of 5-(2-Substituted thiazol-4-yl)-2-pyridones and Thiazolo[4,5-f] quinolinones," Heterocycles, Vol. 29, No. 8, 1989, pp. 1517-1528. doi:10.3987/COM-89-4984

[23] I. Sircar, B. L. Duell, J. A. Bristol, R. E. Weishaar and D. B. Evans, "Cardiotonic Agents. 5. 1,2-Dihydro-5-[4(1Himidazol-1-yl)phenyl]-6-methyl-2-oxo-3-pyridinecarbonitriles and Related Compounds. Synthesis and Inotropic Activity," Journal of Medicinal Chemistry, Vol. 30. No. 6, 1987, pp. 1023-1029. doi:10.1021/jm00389a011

[24] D. W. Robertson, E. E. Beedle, J. K. Swartzendruber, N. D. Jones, T. K. Elzey, R. F. Kauffman, H. Wilson and J. S. Hayes, "Bipyridine Cardiotonics: The Three-Dimensional Structures of Amrinone and Milrinone," Journal of Medicinal Chemistry, Vol. 29, No. 5, 1986, pp. 635-640. doi:10.1021/jm00155a009

[25] J. J. Chen and I. J. Wang, "Synthesis and Colour Assessment of Some 3-Cyano-4-pyrenyl-6-substituted-2-pyridone Derivatives," Dyes and Pigments, Vol. 29, No. 4, 1995, pp. 305-313. doi:10.1016/0143-7208(95)00055-0

[26] A. H. Abadi, T. M. Ibrahim, K. M. Abouzid, J. Lehmann, H. N. Tinsley, B. D. Gary and G. A. Piazza, "Design, Synthesis and Biological Evaluation of Novel Pyridine Derivatives as Anticancer Agents and Phosphodiesterase 3 Inhibitors," Bioorganic \& Medicinal Chemistry, Vol. 17, No. 16, 2009, pp. 5974-5982. doi:10.1016/j.bmc.2009.06.063

[27] D. Villemin, B. Mostefa-Kara, N. Bar, N. ChoukchouBraham, N. Cheikh, A. Benmeddah, H. Hazimeh and C. Ziani-Cherif, "Base Promoted Reaction in Ionic Liquid Solvent: Synthesis of Butenolides," Letters in Organic Chemitry, Vol. 3, 2006, pp. 558-559. doi:10.2174/157017806778341807

[28] D. Villemin, N. Cheikh, B.Mostefa-Kara, N. Bar, N. Choukchou-Braham and M. A. Didi, "Solvent-Free Reaction on KF-Alumina under Microwave: Serendipitous One-Pot Domino Synthesis of New Isobenzofuran-1 $(3 H)$ -ones from Alpha-Hydroxyketones," Tetrahedron Letters,
Vol. 47, No. 31, 2006, pp. 5519-5521. doi:10.1016/j.tetlet.2006.05.137

[29] N. Cheikh, N. Bar, et al., "Efficient Synthesis of New Butenolides by Subsequent Reactions: Application for the Synthesis of Original Iminolactones, Bis-Iminolactones and Bis-Lactones," Tetrahedron, Vol. 67, No. 8, 2011, pp. 1540-1557. doi:10.1016/j.tet.2010.12.062

[30] Q. Shi, J. Chen, Q. Zhunag and X. Wang, "The Condensation of Aromatic Aldehydes with Acidic Methylene Compounds in Water," Chinese Chemical Letters, Vol. 14, 2003, pp. 1242-1245.

[31] T. B. FranColse and F. Andre, "Knoevenagel Condensation Catalysed by Aluminium Oxide," Tetrahedron Letters, Vol. 23, No. 47, 1982, pp. 4927-4928. doi:10.1016/S0040-4039(00)85749-4

[32] P. de la Cruz, E. Diez-Barra, A. Loupy and F. Langa, "Silica Gel Catalysed Knoevenagel Condensation in Dry Media under Microwave Irradiation," Tetrahedron Letters, Vol. 37, 1996, pp. 1113-1116. doi:10.1016/0040-4039(95)02318-6

[33] G.-H. Gao, L. Lu, et al., "Basic Ionic Liquid: A Reusable Catalyst for Knoevenagel Condensation in Aqueous Media," Chemical Research in Chinese Universities, Vol. 23, No. 2, 2007, pp. 169-172. doi:10.1016/S1005-9040(07)60035-X

[34] B. Siebenhaar, B. Casagrande, M. Studer and H. U. Blaser, "An Easy-to-Use Heterogeneous Catalyst for the Knoevenagel Condensation," Canadian Journal of Chemistry, Vol. 79, No. 5-6, 2001, pp. 566-569. doi:10.1139/v01-072

[35] M. L. Kantam and B. Bharathi, "Mn(III) Salen Catalyst for Knoevenagel Condensation a Novel Heterogeneous System," Catalysis Letters, Vol. 55, No. 3-4, 1998, pp. 235-237. doi:10.1023/A:1019051416463

[36] S. Balalaie and N. Nemati, "Ammonium Acetate-Basic Alumina Catalyzed Knoevenagel Condensation under Microwave Irradiation under Solvent-Free Conditions," Synthetic Communications, Vol. 30, No. 5, 2000, pp. 869875. doi:10.1080/00397910008087099

[37] A. McCluskey, P. J. Robinson and T. Hill, "Green Chemistry Approaches to the Knoevenagel Condensation: Comparison of Ethanol, Water and Solvent Free (Dry Grind) Approaches," Tetrahedron Letters, Vol. 43, No. 17, 2002, pp. 3117-3120. doi:10.1016/S0040-4039(02)00480-X

[38] F. Freeman, "Properties and Reactions of Ylidenemalononitriles," Chemical Reviews, Vol. 80, No. 4, 1980, pp. 329-350. doi:10.1021/cr60326a004

[39] A. W. Erian, S. M. Sherif, A. Alassar and Y. M. Elkholy, " $\beta$-Enaminonitriles in Heterocyclic Synthesis: A Novel Synthesis and Transformations of $\alpha$-Substituted- $\beta$-enaminonitriles," Tetrahedron, Vol. 50, No. 6, 1994, pp. 1877 1884. doi:10.1016/S0040-4020(01)80859-0

[40] A. W. Erian, "The Chemistry of $\beta$-Enamino Nitriles as Versatile Reagents in Heterocyclic Synthesis," Chemical Reviews, Vol. 93, No. 6, 1993, pp. 1991-2005. doi:10.1021/cr00022a002 